\title{
Review \\ Hypoxia and oxidative stress in breast cancer Hypoxia and tumourigenesis
}

\author{
Helen J Knowles and Adrian L Harris
}

ICRF Molecular Oncology Laboratory, Weatherall Institute of Molecular Medicine, John Radcliffe Hospital, Oxford, UK

Correspondence: Prof. Adrian L Harris, ICRF, Weatherall Institute of Molecular Medicine, John Radcliffe Hospital, Oxford, OX3 9DS, UK. Tel: + 44 (0)1865 222457; fax: +44 (0)1865 222431; e-mail: aharris.lab@icrf.icnet.uk

Received: 3 May 2001

Accepted: 10 May 2001

Published: 17 July 2001
Breast Cancer Res 2001, 3:318-322

(C) 2001 BioMed Central Ltd

(Print ISSN 1465-5411; Online ISSN 1465-542X)

\begin{abstract}
The microenvironmental hypoxia that arises as a consequence of the development of a solid tumour also acts to promote tumour growth. Hypoxia induces the expression of key components of the angiogenic and apoptotic signalling cascades, the glycolytic pathway and various cell-cycle control proteins. At the cellular level it mediates the infiltration and accumulation of tumour-associated macrophages within avascular tumour regions. Complex interactions between tumour cell and macrophage hypoxia-regulated gene products and their associated pathways form the basis for the hypoxic promotion of tumourigenesis and malignant progression.
\end{abstract}

Keywords: angiogenesis, apoptosis, glycolysis, macrophage

\section{Introduction}

Hypoxia is a feature of solid tumours which generally occurs over $100 \mu \mathrm{m}$ away from functional blood vessels [1], this $\mathrm{O}_{2}$ diffusion distance being determined by metabolism. As human tumours have an inadequate vasculature, hypoxia is widespread in both primary tumours and their metastases [2]. During tumour growth an increasingly compromised blood supply generally results in a tumour oxygen tension of $0-20 \mathrm{mmHg}$, compared with 24-66 mmHg in normal human tissues [3]. The majority of these measurements in human tumours in vivo have been performed using polarographic needle electrodes $[2,3]$ although other less invasive methods, which do not always show good concordance, are at various stages of development. These include magnetic resonance spectroscopy, phosphorescence quenching microscopy and chemical hypoxia probes such as pimonidazole. In addition to chronic diffusion-limited hypoxia tumours can exhibit regions of acute hypoxia, a transient state caused by perfusion fluctuations such as vessel closure, which is also evident in both primary and metastatic human tumour tissue [4]. Factors that have an impact on the rate of $\mathrm{O}_{2}$ utilisation, including the effects of oncogenic transformation on the metabolic rate of tumour tissue, also significantly affect $\mathrm{O}_{2}$ levels [5].

In addition to being a consequence of the growth of a malignant tumour and so a potential marker, hypoxia also acts to promote tumour development. Hypoxic conditions are known to affect the response of solid malignancies to radiation, limiting its efficacy in both primary tumours and metastatic tissue $[6,7]$. As well as conferring resistance to current therapies, hypoxia can act as a negative clinical prognostic indicator. An association between hypoxia and metastatic potential has been demonstrated in human tumours $[6,8]$ with tumour oxygenation being a strong predictor of both overall and disease-free survival, regardless of the mode of primary treatment [8]. The clinical impact of hypoxia is supported by the results of trials suggesting beneficial effects of blood transfusion or infusion of recombinant human erythropoietin on treatment outcome, particularly in response to radiotherapy [9]. 


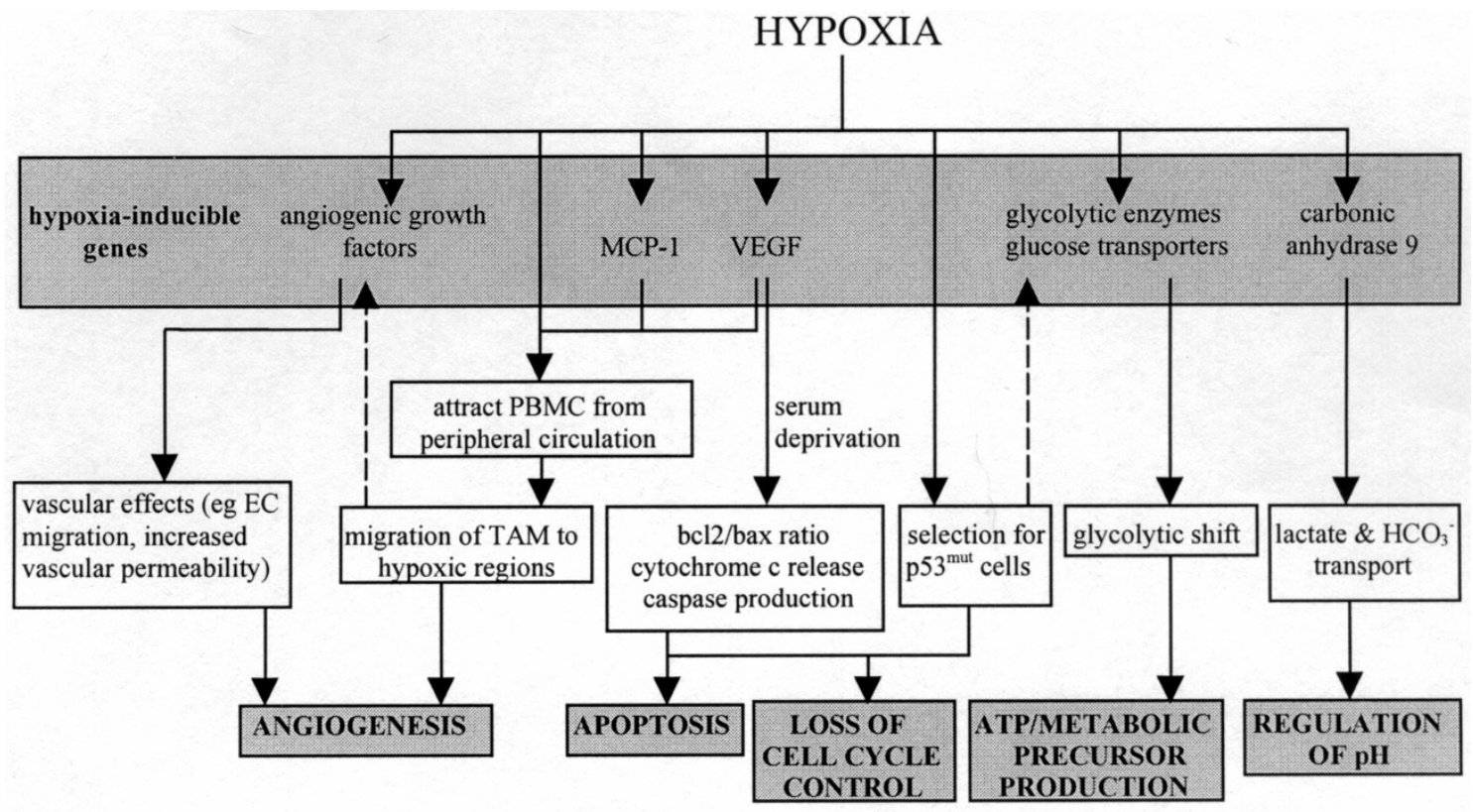

Hypoxic regulation of pathways promoting tumour growth. The transcriptional level response to hypoxia is amplified by positive feedback mechanisms and substantial crosstalk between the interconnecting pathways, thus providing a strong, co-ordinated response promoting tumourigenesis and malignant progression. EC, endothelial cell; MCP-1, monocyte chemotactic protein 1; PBMC, peripheral blood mononuclear cell; TAM, tumour-associated macrophage; VEGF, vascular endothelial growth factor.

The basis for the association between hypoxia, tumourigenesis and malignant progression has been examined extensively at both the molecular and the cellular level. Hypoxia exerts direct effects on the expression of numerous gene products involved in processes such as angiogenesis, apoptosis, glycolysis and cell-cycle control, which are central to the survival and expansion of a malignant cell population in an oxygen-deficient environment. Hypoxia is also known to mediate the recruitment of macrophages and other immune cells into tumour tissue. These cells, in turn, play a critical role in the balance between tumour regression and progressive malignancy (Fig. 1).

\section{Tumour angiogenesis}

The main mechanism whereby a solid tumour mass can overcome the proliferative limitations imposed by stressful microenvironmental conditions and thus expand beyond the microscopic level is to develop a nutritive blood supply. This requires a switch from the constitutive anti-angiogenic phenotype, involving a change in the balance of pro- and anti-angiogenic molecules secreted from tumours and surrounding cells, brought about by a combination of genetic and microenvironmental stimuli, including hypoxia. Vascular endothelial growth factor (VEGF) is a major pro-angiogenic molecule that is mitogenic for endothelial cells and acts to increase the vascular permeability of tumour blood vessels. VEGF and one of its cellular receptors (flk-1) are significantly upregulated in response to hypoxia $[10,11]$, as are a variety of other pro-angiogenic growth factors and their receptors including platelet-derived growth factor-BB, endothelin, nitric oxide synthase, insulin-dependent growth factor-II, adrenomedullin and epidermal growth factor receptor [11]. Oncogenic transformation of cells with activated ras has been shown to increase both the basal level of VEGF mRNA expression and its degree of induction under hypoxia. This is also true of other oncogenes including egfr, erbB2 and raf, demonstrating that activation of oncogene pathways can also switch on the angiogenic phenotype [12].

As well as providing a physiological stimulus for the de novo formation of tumour blood vessels, systemic hypoxia is known to increase oxygen delivery to tumour tissues by stimulating erythropoietin production. It was as a result of the search for the molecular basis of this upregulation that the hypoxia inducible factor (HIF) transcription factor was discovered. HIF-1 (a heterodimeric transcription factor composed of a hypoxia-inducible $\alpha$ subunit and a constitutive $\beta$ subunit) has been directly implicated in the hypoxic upregulation of a number of the angiogenic growth factors, including VEGF. The importance of the downstream effects of the HIF-mediated molecular response to hypoxia on tumour growth has been dramatically demonstrated in vivo. Abolition of the angiogenic growth factor response to hypoxia in HIF-1 $\alpha^{-/-}$embryonic stem cells and Hepa1 cells lacking the aryl hydrocarbon receptor 
nuclear translocator has generally resulted in xenografts that demonstrate reduced vascularity compared to their wild-type counterparts when grown as solid tumours in vivo $[13,14]$. In a more recent study HIF-1 $\alpha^{-/-}$tumours exhibited no significant reduction in vascularity compared with tumours derived from wild-type cells, despite a reduction in growth rate [15], although this discrepancy may be cell-line specific. Generally, however, loss of the hypoxiastimulated angiogenic growth factor response has demonstrated the importance of tumour vascularisation to the growth and expansion of solid malignant tumours.

\section{Apoptosis}

As hypoxia is present prior to new blood vessel formation, it is vital that tumour cells are able to adapt to a nutrientdeficient environment and survive for long enough periods for tumour progression to occur. It has recently been shown that hypoxia is able to suppress the proapoptotic effects of serum deprivation on both endothelial cells and tumour cells via induction of VEGF $[16,17]$. In HepG2 cells hypoxia inhibited apoptosis, reduced the bax:bcl-2 ratio, decreased cytochrome $\mathrm{c}$ release and decreased caspase 3 activity. These effects were blocked by anti-VEGF neutralising antibodies or inhibitors of receptor tyrosine kinase signalling [17]. This rescuing effect of hypoxia could be mimicked by the addition of recombinant human VEGF to serum-deprived cells. These data suggest that the hypoxic induction of VEGF during serum deprivation could be an adaptive response, making tumour cells less susceptible to apoptosis-inducing stimuli prior to initiation of new blood vessel formation [17]. Although the role of VEGF receptors and their degree of expression in tumour cell lines versus endothelium is poorly studied, these results suggest that a more detailed evaluation is warranted.

Another mechanism by which hypoxia subverts normal mechanisms of apoptosis was described by Graeber et al who demonstrated how hypoxia can act as a physiological selective pressure promoting the clonal expansion of cells with acquired mutations in components of the apoptotic pathway [18]. Exposure of oncogenically-transformed mouse embryonic fibroblast cells to multiple rounds of hypoxia and aerobic recovery revealed the ability of hypoxia to select for $p 53^{-/-}$(apoptosis resistant) over $p 53^{\mathrm{WT}}$ cells. This in vitro data was supported by the spatial relationship between hypoxia, p53 and apoptosis in murine tumours in vivo, suggesting that hypoxia can promote the clonal expansion of $p 53^{\text {mut }}$ cells in areas of tumour tissue where other cells will be killed by apoptosis [18]. The relevance of these findings to human tumourigenesis is supported by data indicating that loss of p53 $\mathrm{WT}$ is associated with elevated levels of the apoptosis inhibitor bcl-2, and the general observation that apoptosis is inhibited during the development of human cancer $[19,20]$. This reduced $\mathrm{p} 53: \mathrm{bcl}-2$ ratio (indicative of loss of both cell-cycle control and regulation of the major apoptotic pathway) acts to increase mutation rates within a clonal cell population, aiding tumourigenesis and expansion of solid malignancies. Passive selection of apoptosis-resistant cells by a component of the tumour's own microenvironment could help to explain why $p 53$ is the most commonly mutated gene in human cancer.

Importantly, it is not only anti-apoptotic pathways that are selected under hypoxic conditions. Expression of the cell death factor NIP3 is known to be upregulated by hypoxia in a HIF-dependent manner in a variety of cancer cell lines [21] and human tumours [22]. Large-scale gene expression studies have identified a number of pro-apoptotic or growth-inhibitory genes that are specifically upregulated under hypoxia, as well as those that promote tumour growth [23]. It may be the case, therefore, that co-ordinated regulation of a number a pro- and anti-apoptotic pathways by both HIF-dependent and HIF-independent mechanisms governs susceptibility to hypoxia-induced apoptosis in a cell-type-specific manner.

\section{The glycolytic shift}

As insufficient oxygen is available for efficient ATP production via the electron transport chain under hypoxic conditions, another major cellular adaptation to hypoxia is the switch of the respiratory pathway to anaerobic glycolysis. This is associated with the high levels of glucose consumption characteristic of solid tumour tissue [3] and is necessary in order to maintain both the supply of precursors of anabolic pathways (lipids, amino acids, nucleic acids etc.) and cellular levels of ATP production within this stressful environment.

Metabolic adaptation to tumour microenvironmental conditions is a crucial step in tumour progression and hypoxia acts to maximise the efficiency of the glycolytic shift on several levels. Firstly, the expression of many key glycolytic enzymes (including phosphoglycerate kinase 1, lactate dehydrogenase $A$, pyruvate kinase $M$, glyceraldehyde-3phosphate dehydrogenase, enolase 1 and aldolase A) is coordinately upregulated under hypoxia [24]. Secondly, over-expression of the glucose transporter genes glut1 and glut3 is also known to be regulated by hypoxia and has been demonstrated in human tumour tissues [13]. Thirdly, hypoxia can contribute more indirectly to the glycolytic phenotype; hypoxically-selected mutant p53 is able to specifically transactivate glycolytic enzymes such as the type II isoform of hexokinase [25]. The effects of hypoxia can thus provide a direct link between the loss of cellcycle control and the high glycolytic rate of tumour cells. Taken together, these gene expression changes enable both maximal glucose uptake and a high efficiency of glucose utilisation. Tumour cells, therefore, are able to undergo glycolytic respiration, grow and proliferate under more severe conditions than possible for normal cells. 


\section{The contribution of tumour-associated macrophages}

As well as modulating tumour biology at the molecular level, hypoxia exerts cellular level effects on solid tumours. Hypoxia is known to induce transendothelial migration of monocytic cells from the peripheral circulation, which then migrate into tumour tissue along gradients of chemokines such as monocyte chemotactic protein 1 [26]. This migration is inhibited when a region of hypoxia is encountered and the cells are then induced to accumulate and function as tumour-associated macrophages (TAMs) [27]. This process, therefore, acts to direct macrophages to areas of necrosis or hypoxia where they exhibit a tumourigenic phenotype. Such macrophages have been associated with angiogenesis and poor prognosis in invasive breast carcinoma [28]. The molecular basis of the pro-angiogenic activity of TAMs is similar to that of tumour cells themselves in that hypoxia induces the expression of a variety of cytokines and growth factors. A key growth factor is VEGF, which is thought to act on macrophages via a positive feedback mechanism. Hypoxia stimulates the production of VEGF by both TAMs and tumour cells and it then exerts a chemotactic action on macrophages, aiding their migration to avascular tumour sites [29]. Macrophage-produced VEGF may then act in the angiogenic and antiapoptotic manner already discussed in order to further the malignant phenotype.

\section{Conclusions}

It is clear that in addition to being a well-documented marker of solid human tumour tissue, hypoxia also acts on several levels to promote further tumour development. We have highlighted mechanisms whereby hypoxia mediates the switch to a pro-angiogenic phenotype, subverts normal apoptotic pathways and maintains high levels of metabolic activity within this stressful microenvironment. Using VEGF as an example, we have also demonstrated the complex interrelationships between these pathways. VEGF is a major pro-angiogenic molecule that suppresses the proapoptotic effects of serum deprivation under hypoxia and contributes to the accumulation of TAMs in avascular tumour sites, which then produce further VEGF as part of a positive feedback mechanism. Antagonism of VEGF, therefore, may have multiple pathways of interaction with other therapies, as well as direct antitumour effects.

It should be stressed that the genes discussed in this review constitute only a fraction of those that have been identified as being regulated by hypoxia. Gene expression studies have defined numerous growth factors, glycolytic enzymes, cytokines, transcription factors etc., which are inducible by hypoxia, either by HIF-dependent or by HIFindependent pathways. Many of these genes will act on the major pathways that have been discussed while others will have more distinct actions, for example the HIFinducible carbonic anhydrase 9 which might exert its bio- logical effect through regulation of microenvironmental $\mathrm{pH}$ [30]. As extracellular acidosis is also thought to promote tumour growth, this adds yet another dimension to the already complex nature of the contribution of hypoxia to tumourigenesis.

\section{References}

1. Helmlinger G, Yuan F, Dellian $M$, Jain RK: Interstitial $\mathbf{p H}$ and $\mathbf{p O}_{2}$ gradients in solid tumours in vivo: high resolution measurements reveal a lack of correlation. Nat Med 1997, 3:177-182.

2. Brizel DM, Rosner GL, Prosnitz LR, Dewhirst MW: Patterns and variability of tumour oxygenation in human soft tissue sarcomas, cervical carcinomas and lymph node metastases. Int $J$ Radiat Oncol Biol Phys 1995, 32:1121-1125.

3. Vaupel P, Kallinowski F, Okunieff P: Blood flow, oxygen and nutrient supply, and metabolic microenvironment of human tumours: a review. Cancer Res 1989, 49:6449-6465.

4. Hill SA, Pigott KH, Saunders MI, Powell MEB, Arnold S, Obeid A, Ward G, Leahy M, Hoskin PJ, Chaplin DJ: Microregional blood flow in murine and human tumours assessed using laser Doppler microprobes. Br J Cancer 1996, 74:S260-S263.

5. Cohen-Jonathan E, Evans SM, Koch CJ, Muschel RJ, McKenna G, Wu JM, Bernhard EJ: The farnesyltransferase inhibitor L744,832 reduces hypoxia in tumours expressing activated $\mathrm{H}$ ras. Cancer Res 2001, 61:2289-2293.

6. Brizel DM, Scully SP, Harrelson JM, Layfield LJ, Beau JM, Prosnitz LR, Dewhirst MW: Tumour oxygenation predicts for the likelihood of distant metastases in human soft tissue sarcoma. Cancer Res 1996, 56:941-943.

7. Gatenby RA, Kessler HB, Rosenblum JS, Coia LR, Moldofsky PJ, Hartz WH, Broder GJ: Oxygen distribution in squamous cell carcinoma metastases and its relation to outcome of radiation therapy. Int J Radiat Oncol Biol Phys 1988, 14:831-838.

8. Hockel M, Schlenger K, Aral B, Mitze M, Schaffer U, Vaupek P: Association between tumour hypoxia and malignant progression in advanced cancer of the uterine cervix. Cancer Res 1996, 56:4509-4515.

9. Dicato M, Duham C, Berchem G, Ries F: Clinical benefit from erythropoietin. Curr Opin Oncol 2000, 12:297-302.

10. Shweiki D, Itin A, Soffer D, Keshet E: Vascular endothelial growth factor induced by hypoxia may mediate hypoxia-initiated angiogenesis. Nature 1992, 359:843-845.

11. Carmeliet $P:$ Mechanisms of angiogenesis and arteriogenesis. Nat Med 2000, 6:389-395.

12. Rak J, Yu JL, Klement G \& Kerbal RS: Oncogenes and angiogenesis: signaling three-dimensional tumour growth. J Invest Dermatol Symp Proc 2000, 5:24-33.

13. Maxwell PH, Dachs GU, Gleadle JM, Hicholls LG, Harris AL, Stratford IJ, Hankinson O, Pugh CW, Ratcliffe PJ: Hypoxiainducible factor-1 modulates gene expression in solid tumours and influences both angiogenesis and tumour growth. Proc Natl Acad Sci USA 1997, 94:8104-8109.

14. Ryan HE, Lo J, Johnson RS: HIF- $1 \alpha$ is required for solid tumour formation and embryonic vascularisation. EMBO J 1998, 17: 3005-3015.

15. Ryan HE, Poloni M, McNulty W, Elson D, Gassmann M, Arbeit M, Johnson RS: Hypoxia-inducible factor- $1 \alpha$ is a positive factor in solid tumour growth. Cancer Res 2000, 60:4010-4015.

16. Gupta K, Kshirsagar S, Li W, Gui W, Ramakrishnan S, Gupta P, Law PY, Hebbel RP: VEGF prevents apoptosis of human microvascular endothelial cells via opposing effects on MAPK/ERK and SAPK/JNK signalling. Exp Cell Res 2000, 247: 495-504.

17. Baek JH, Jang J-E, Kang C-M, Chung H-Y, Kim ND, Kim K-W: Hypoxia-induced VEGF enhances tumour survivability via suppression of serum deprivation-induced apoptosis. Oncogene 2000, 19:4621-4631.

18. Graeber TG, Osmanian C, Jacks T, Housman DE, Koch CJ, Lowe SW, Giaccia AJ: Hypoxia-mediated selection of cells with diminished apoptotic potential in solid tumours. Nature 1996, 379:88-91.

19. Haldar S, Negrini M, Monne M, Sabbioni S, Croce CM: Downregulation of bcl-2 by p53 in breast cancer cells. Cancer Res 1994, 54:2095-2097. 
20. Bedi A, Pasricha PJ, Akhtar AJ, Barber JP, Bedi GC, Giardiello FM, Zehnbauer BA, Hamilton SR, Jones RJ: Inhibition of apoptosis during development of colorectal cancer. Cancer Res 1995, 55:1811-1816.

21. Bruick RK: Expression of the gene encoding the proapoptotic Nip3 protein is induced by hypoxia. Proc Natl Acad Sci USA 2000, 97:9082-9087.

22. Sowter HM, Ratcliffe PJ, Watson $P$, Greenberg $A H$, Harris AL: HIF-1-dependent regulation of hypoxic induction of the cell death factors BNIP3 and NIX in human tumours. Cancer Research 2001, in press.

23. Wykoff CC, Pugh CW, Maxwell PH, Harris AL \& Ratcliffe PJ: Identification of novel hypoxia dependent and independent target genes of the von Hippel-Lindau (VHL) tumour suppressor by mRNA differential expression profiling. Oncogene 2000, 19:6297-6305.

24. Semenza GL, Roth PH, Fang H-M, Wang GL: Transcriptional regulation of genes encoding glycolytic enzymes by hypoxiainducible factor 1. J Biol Chem 1994, 269:23757-23763.

25. Mathupala SP, Heese C, Pederson PL: Glucose catabolism in cancer cells. J Biol Chem 1997, 36:22776-22780.

26. Kalra VK, Shen Y, Sultana C, Rattan V: Hypoxia induces PECAM-1 phosphorylation and transendothelial migration of monocytes. Am J Physiol 1996, 271:H2025-H2034.

27. Turner L, Scotton $C$, Negus $R$, Balkwill $F$ : Hypoxia inhibits macrophage migration. Eur J Immunol 1999, 29:2280-2287.

28. Leek RD, Lewis CE, Whitehouse R, Greenall M, Clarke J, Harris AL: Association of macrophage infiltration with angiogensis and prognosis in invasive breast carcinoma. Cancer Res 1996, 56:4625-4629.

29. Lewis JS, Landers RJ, Underwood JC, Harris AL, Lewis CE: Expression of vascular endothelial growth factor by macrophages is upregulated in poorly vascularised areas of breast cancers. J Pathol 2000, 192:150-158.

30. Wycoff CC, Beasley NJP, Watson PH, Turner KJ, Pastorek J, Sibtain A, Wilson GD, Turley H, Talks KL, Maxwell PH, Pugh C, Ratcliffe PJ, Harris AL: Hypoxia-inducible expression of tumour-associated carbonic anhydrases. Cancer Res 2000, 60:7075-7083. 